Notre Dame Journal of Formal Logic

Volume XIV, Number 1, January 1973

NDJFAM

\title{
REMARK ABOUT THE BOOLEAN PARTS IN THE POSTULATE-SYSTEMS OF CLOSURE, DERIVATIVE AND PROJECTIVE ALGEBRAS
}

\author{
BOLESŁAW SOBOCIŃNKI
}

1 The contents of this note are strictly connected with the results which have been proved in [7]. ${ }^{1}$ Therefore, in order to present the discussions which will be given below in a uniform and similar way to that which can be found in [7], and, moreover, in order to avoid unnecessary and repetitious explanations it is accepted throughout this paper that

(a) the formalizations of Boolean algebras, non-associative Newman algebras and the dual non-associative Newman algebras which will be used below are exactly the same, as given in the points (B), (D), and (F) respectively in [7], pp. 530, 535 and 539 ,

and, furthermore, that

(b) the definitions of the algebraic systems under discussion in this note will be formulated in accordance with the points (a), (b) and (c) given in [7], p. 531.

It should be noted that the formalizations accepted in point (a) of any algebras mentioned above can be substituted by an arbitrary formalization providing that it is equivalent to the former without affecting the results which will be presented below. Clearly, these two clauses cannot lead to any misunderstanding.

1.1 If a postulate-system of the given algebras which are Boolean algebras extended by the additional extra-Boolean operations and postulates contains the following postulate:

Co the structure $\langle A,+, \times,-, 0,1\rangle$ is a Boolean algebra

1. An acquaintance with [7] is presupposed. Especially, in order to understand the proofs given in sections 2,3 and 4 below a certain familiarity with the systems $\mathfrak{M}$ and $\mathfrak{N}$, $c f$. [7], pp. 531-539, is required. 
then such postulate is called the Boolean part of these algebras, $c f .[3]$, pp. 162-163, definition 1.1.1 and pp. 430-431, definition 2.7.1. In [7], p. 525, Theorem I, and pp. 540-541, Theorem II, it has been proved that in the postulate system of a cylindric algebra of dimension $\alpha \geq 1$ given in [3], pp. 161-163, its Boolean part, i.e., the postulate $C O$ can be replaced by one of the following postulates:

C $0^{*}$ the structure $\langle A,+, \times,-, 0,1\rangle$ is a non-associative Newman algebra

or

CO** the structure $\langle A,+, \times,-, 0,1\rangle$ is a dual non-associative Newman algebra

each of which is weaker, $c f$. [7], pp. 541-545, section 3, than $C O$.

A natural question arises whether in the postulate-systems containing Boolean parts of the other algebras (apart from the cylindric algebras) it is possible to replace $C O$ by weaker assumptions without the change of their extra-Boolean postulates. It will be shown below that in the well-known postulate-systems of closure, derivative and projective algebras it holds, i.e., that the assumptions that each of these postulate-systems contain the Boolean part are stronger than needed.

2 In accordance with the points (a) and (b) given in section 1 we formalize the closure algebras defined, e.g., in [5], pp. 145-146, and in [4], p. 931, definition 3.11 , as follows:

(I) Any algebraic structure

$$
\boldsymbol{c}=\langle A,+, \times,-, 0,1, \mathrm{C}\rangle
$$

where + and $\times$ are two binary operations, and - and $C$ are two unary operations defined on the carrier set $A$, and 0 and 1 are two constant elements belonging to $A$, is a closure algebra, if it satisfies the following postulates: $C O$ and

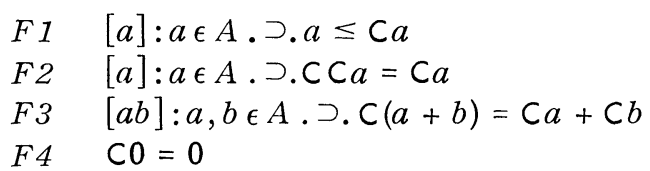

Since in $C$ we have $C O$ and " $\leq$ " is not a primitive notion of the investigated system, obviously we have two inferentially equivalent forms of $F 1$, viz.

$F 1^{*}[a]: a \epsilon A . \supset . a+\mathrm{C} a=\mathrm{C} a$

and

$F 1^{* *}[a]: a \in A . \supset . a \times \mathrm{C} a=a$

Therefore, we have two versions which are equivalent to the postulatesystem of $\mathbb{C}$, viz. $\left\{C O, F 1^{*}, F 2, F 3, F 4\right\}$ and $\left\{C O, F 1^{* *}, F 2, F 3, F 4\right\}$. We shall prove: 
$(\alpha)$ In the postulate systems $\left\{C O, F 1^{*}, F 2, F 3, F 4\right\}$ and $\left\{C O, F 1^{* *}, F 2, F 3\right.$, $F 4\}$ the postulate $C O$ can be replaced by $C 0^{*}$ and $C O^{* *}$ respectively.

Proof: (i) Assume $F I^{*}$ and the system $\mathfrak{M}$. Hence, due to $\mathfrak{M}$ we have $M 1$, $M 4, D 1, M 7$ and M25, cf. [7], pp. 532-533. Then:

$R 1 \quad[a]: a \in A . \supset . a=a+a$

PR $[a]: \mathrm{Hp}(1) . \supset$.

$$
\begin{aligned}
a & =a \times(\mathrm{C} 1+-\mathrm{C} 1)=a \times((1+\mathrm{C} 1)+-\mathrm{C} 1) \\
& =a \times(1+(\mathrm{C} 1+-\mathrm{C} 1))=a \times(1+1)=a+a
\end{aligned}
$$

$[M 25 ; D 1 ; M 1 ; M 7]$

(ii) Now, assume $F 1^{* *}, F 4$ and the system $\mathfrak{R}$. Hence, due to $\mathfrak{R}$ we have $N 1$ and $N 7, c f$. [7], pp. 536-537. Then:

TI $[a]: a \in A . \supset . a=a \times a$

PR $[a]: \operatorname{Hp~(1).\supset .~}$

$$
a=a+0=a+(0 \times \mathrm{C} 0)=a+(0 \times 0)=a \times a \quad\left[1 ; N 7 ; F 1^{* *} ; F 4 ; N 1 ; N 7\right]
$$

Since the addition of $R 1$ and $T 1$, as the new postulates to the systems $\mathfrak{M}$ and $\mathfrak{N}$ respectively yield Boolean algebra in both cases, $c f$. [7], pp. 533534 , section 1.2, and pp. 538-539, section 2.2, the proof of $(\alpha)$ is complete. Thus, in the postulate-system of closure algebras the postulate $C O$ is stronger than needed.

2.1 It is well-known that the so-called interior operation defined in the field of closure algebras:

$$
\mathrm{I} a=-\mathrm{C}-a
$$

can serve, as the primitive notion of these algebras. A postulate-system based on 1 of closure algebras is given, e.g., in [6], p. 94, and it is formalized here, as follows:

(II) Any algebraic structure

$$
\boldsymbol{c}=\langle A,+, \times,-, 0,1,1\rangle
$$

where,$+ \times,-, 0$ and 1 are defined, as in (I), and $\mathrm{I}$ is a unary operation defined on the carrier set $A$, is a closure algebra, if it satisfies the following postulates: $\mathrm{C} O$ and

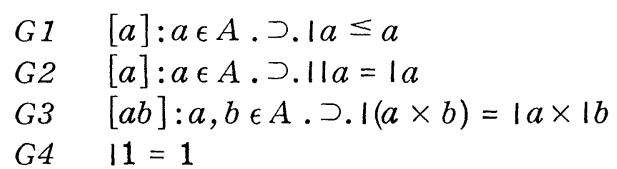

Again due to the availability of $C O$ we have two inferentially equivalent forms of $G 1$, viz.:

$G 1^{*}[a]: a \in A . \supset .1 a+a=a$

and

$G 1^{* *}[a]: a \in A . \supset . \mid a \times a=1 a$ 
We shall prove:

(B) In the postulate systems $\left\{C O, G 1^{*}, G 2, G 3, G 4\right\}$ and $\left\{C O, G 1^{* *}, G 2, G 3\right.$, $\left.{ }_{G 4}\right\}$ the postulate $\mathrm{CO}$ can be replaced by $\mathrm{CO}$ * and $\mathrm{CO} \mathrm{O}^{* *}$ respectively.

Proof: (i) Assume $G 1^{*}, G 4$ and the system $\mathfrak{M}$. Hence, due to $\mathfrak{M}$ we have $M 1$ and $M 7, c f$. section 2 above. Then:

$R 1 \quad[a]: a \in A . \supset . a=a+a$

PR $[a]: \operatorname{Hp}(1) . \supset$.

$a=a \times 1=a \times(\mid 1+1)=a \times(1+1)=a+a \quad\left[1 ; M 7 ; G 1^{*} ; G 4 ; M 1 ; M 7\right]$

(ii) Assume $G 1^{* *}, G 2$ and the system $\mathfrak{N}$. Hence, due to $\mathfrak{N}$ we have $N 1, D f 1$, $N 7$ and $N 25$, cf. [7], pp. 536-538. Then:

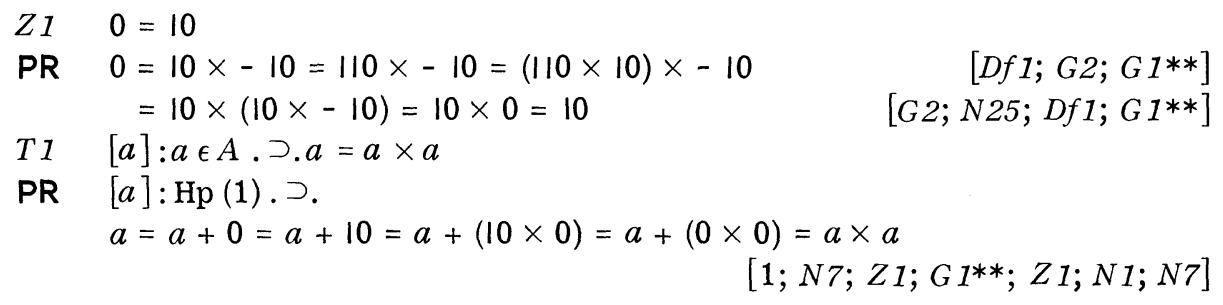

Thus, $c f$. section 2 above, the proof of $(\beta)$ is complete, and, therefore, in the formalization (II) of closure algebras the postulate $C O$ is stronger than needed.

3 The derivative algebras and the dense-in-itself derivative algebras are formulated and defined in [5], pp. 182-184. Here, they are formalized, as follows:

(III) Any algebraic structure

$$
\mathfrak{D}=\langle A,+, \times,-, 0,1, \mathrm{D}\rangle
$$

where,$+ \times,-, 0$ and 1 are defined, as in (I), and $\mathrm{D}$ is a unary operation defined on the carrier set $A$, is a derivative algebra, if it satisfies the following postulates: $C O$ and

$H 1 \quad[a]: a \epsilon A . \supset . \mathrm{DD} a \leq \mathrm{D} a$

H2 $[a b]: a, b \in A . \supset . \mathrm{D}(a+b)=\mathrm{D} a+\mathrm{D} b$

H3 $\mathrm{DO}=0$

Moreover, if besides $\mathrm{CO}, \mathrm{H1}, \mathrm{H} 2$ and $\mathrm{H} 3, \mathfrak{D}$ satisfies an additional postulate

H4 D $1=1$

then the structure $\mathfrak{D}$ is a dense-in-itself derivative algebra.

Once more, due to $C O$ we have two inferentially equivalent forms of the axiom $H 1$, viz.:

$H 1^{*}[a]: a \epsilon A . \supset . \mathrm{D} a+\mathrm{D} a=\mathrm{D} a$

and 
$H 1^{* *}[a]: a \in A . \supset . \mathrm{DD} a \times \mathrm{D} a=\mathrm{DD} a$

3.1 I have been unable to prove that in $\left\{\mathrm{CO}, \mathrm{H} 1^{*}, \mathrm{H} 2, \mathrm{H} 3\right\}$ the postulate $\mathrm{CO}$ can be replaced by $C 0^{*}$, but it can be proved that:

$(\gamma)$ In the postulate-system $\left\{\mathrm{CO}, \mathrm{H}^{* *}, \mathrm{H} 2, \mathrm{H} 3\right\}$ of derivative algebras the postulate $C O$ can be replaced by CO**.

Proof: Assume $H 1^{* *}, H 3$ and the system $\mathfrak{R}$. Hence, due to $\mathfrak{R}$ we have $N 1$ and $N 7, c f$. section 2 above. Then:

$Z 1 \quad \mathrm{DD} 0=\mathrm{D} 0$

T1 [a]: $a \in A . \supset . a=a \times a$

PR $[a]: \operatorname{Hp}(1) . \supset$.

$a=a+0=a+\mathrm{DO}=a+\mathrm{DDO}=a+(\mathrm{DDO} \times \mathrm{D} 0) \quad\left[1 ; N 7 ; H 3 ; Z 1 ; H 1^{* *}\right]$

$=a+(0 \times 0)=a \times a$

$[Z 1 ; H 3 ; N 1 ; N 7]$

Thus, $c f$. section 2, the proof of $(\gamma)$ is complete, which shows that in formalization (III) of derivative algebras the postulate $C O$ is stronger than needed.

3.2 In the field of dense-in-itself derivative algebras not only $(\gamma)$ holds obviously, but also:

(ס) In the postulate system $\left\{\mathrm{CO}, \mathrm{H}^{*}, \mathrm{H} 2, \mathrm{H} 3, \mathrm{H} 4\right\}$ of dense-in-itself derivative algebras the postulate $C O$ can be replaced by $C O *$.

Proof: Assume $H 1^{*}, H 4$ and the system $\mathfrak{M}$. Hence, due to $\mathfrak{M}$ we have $M 1$, $M 4, M 7$ and $M 25, c f$. section 2. Then:

$Z 1 \quad \mathrm{DD} 1=\mathrm{D} 1$

$[H 4]$

$R 1 \quad[a]: a \in A . \supset . a=a+a$

PR $[a]: H p(1) . \supset$.

$$
\begin{aligned}
a & =a \times(\mathrm{D} 1+-\mathrm{D} 1)=a \times((\mathrm{DD} 1+\mathrm{D} 1)+-\mathrm{D} 1) \\
& =a \times(\mathrm{DD} 1+(\mathrm{D} 1+-\mathrm{D} 1))=a \times(1+1)=a+a
\end{aligned}
$$

$[M 25 ; Z 1 ; H 4 ; D 1 ; M 1 ; M 7]$

Thus, $c f$. section 2, the proof of $(\delta)$ is complete.

4 There are two equivalent formalizations of projective algebras. The first is given in [2], where Everett and Ulam formulated and investigated the theory of these algebras. The second formalization which is much simpler and shorter than the first, is announced by Chin and Tarski in [1], and its final corrected form is presented in [4], pp. 938-939. For our purpose we shall investigate only the version given in [4] which can be defined as follows:

(IV) Any algebraic structure

$$
\mathfrak{B}=\left\langle A,+, \times,-, 0,1, \mathbf{c}_{0}, \mathbf{c}_{1}, \theta\right\rangle
$$

where,$+ \times,-, 0$ and 1 are defined, as in (I), $\mathbf{c}_{0}$ and $\mathbf{c}_{1}$ are two unary operations defined on the carrier set $A$ and $\theta$ is a constant element belonging to $A$, is a projective algebra, if it satisfies the following postulates: $C O$ and 


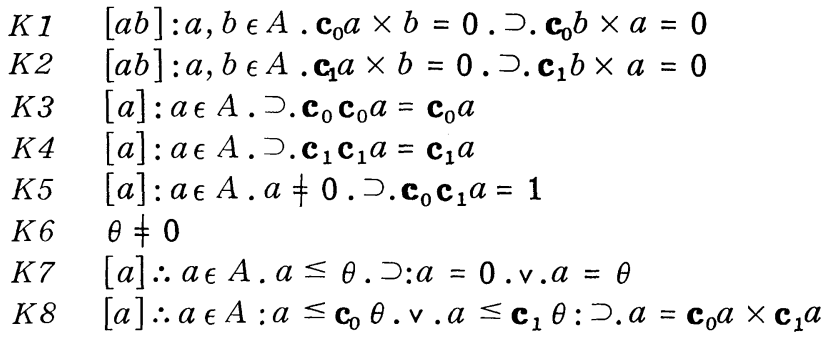

Clearly, due to $K 6$ and $K 7, \theta$ is an atom. Moreover, $c f$. [4], p. 938, and [3], p. 288, Theorem 2.3.14, we know that:

If the substructure $\left\langle A,+, \times,-, 0,1, \mathbf{c}_{0}, \mathbf{c}_{1}\right\rangle$ of $\mathfrak{B}$ satisfies the postulates CO, K1-K5, then it is a simple diagonal-free cylindric algebra of dimension 2.

I have been unable to prove that in $\{C O, K 1, K 2, K 3, K 4, K 5\}$ the postulate $C O$ can be replaced either by $C 0^{*}$ or by $C O * *$, and that in $\{C O, K 1$, $K 2, K 3, K 4, K 5, K 6, K 7, K 8\}$ the postulate $C O$ can be replaced by $C 0^{*}$, but we have:

(ع) In the postulate-system $\{C O, K 1, K 2, K 3, K 4, K 5, K 6, K 7, K 8\}$ of projective algebras the postulate $C O$ can be replaced by $C 0^{* *}$ regardless whether an abbreviation " $a \leq b$ " is understood as " $a+b=b$ " or as " $a \times b=a$."

Proof: Assume $K 1, K 2, K 3, K 4, K 5, K 6, K 8$ and the system $\mathfrak{R}$. Hence, due to $\mathfrak{R}$ we have $N 1, N 7, N 8, N 14, N 19$ and N24, cf. [7], pp. 536-538. Then:

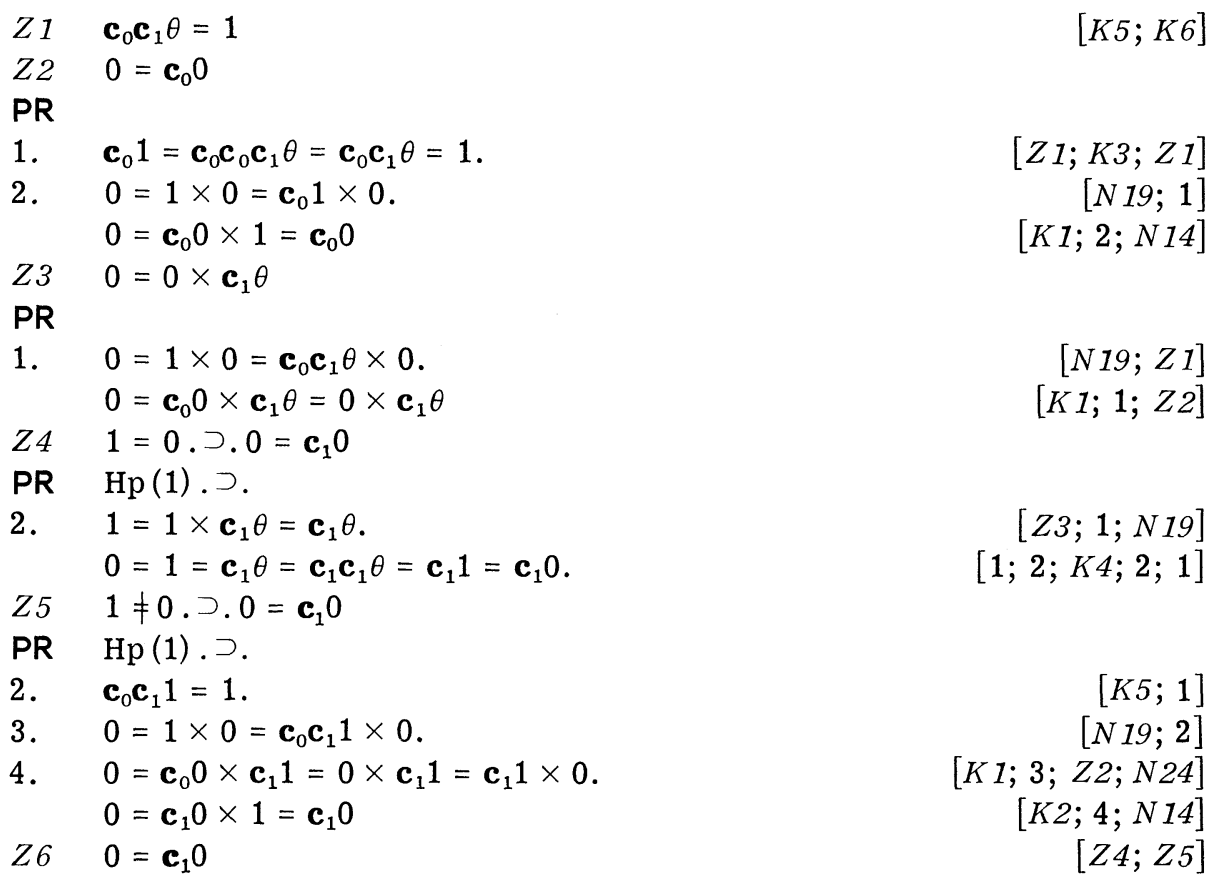


Since we have at our disposal $N 8$ and $Z 3$, it is indifferent whether a formula " $0 \leq \mathbf{c}_{1} \theta$ " will be considered as an abbreviation of " $0+\mathbf{c}_{1} \theta=\mathbf{c}_{1} \theta$ " or of " $0 \times \mathbf{c}_{1} \theta=0$ ". Whence:

$Z 7 \quad 0=0 \times 0$

PR $0=\mathbf{c}_{0} 0 \times \mathbf{c}_{1} 0=0 \times 0$

T1 $[a]: a \in A . \supset . a=a \times a$

$[K 8 ; Z 3$ or $N 8 ; Z 2 ; Z 6]$

PR $\quad[a]: \operatorname{Hp}(1) . \supset$.

$$
a=a+0=a+(0 \times 0)=a \times a
$$

Thus, $c f$. section 2, the proof of $(\varepsilon)$ is complete which shows that in the formalization (IV) of the projective algebras the postulate $C O$ is stronger than needed.

Remark: It is easy to prove that if, instead of postulate $K 8$, we accept

$K 8^{*} \quad[a] \therefore a \varepsilon A: a \leq \mathbf{c}_{0} \theta \cdot \vee . a \leq \mathbf{c}_{1} \theta: \supset .-a=-\mathbf{c}_{0} a+-\mathbf{c}_{1} a$

which in the field of $C O$ is obviously inferentially equivalent to $K 8$, then in $\left\{C O, K 1, K 2, K 3, K 4, K 5, K 6, K 7, K 8^{*}\right\}$ the postulate $C O$ can be replaced by $C O^{*}$.

\section{REFERENCES}

[1] Chin, L. H., and A. Tarski, "Remarks on projective algebras," Bulletin of the American Mathematical Society, vol. 54 (1948), pp. 80-81.

[2] Everett, C. J., and S. Ulam, "Projective algebras I," American Journal of Mathematics, vol. 68 (1946), pp. 77-88.

[3] Henkin, L., J. D. Monk, and A. Tarski, Cylindric Algebras, Part I, North-Holland Publishing Company, Amsterdam-London (1971).

[4] Jónsson, B., and A. Tarski, "Boolean algebras with operators. Part I," American Journal of Mathematics, vol. 73 (1951), pp. 891-939.

[5] McKinsey, J. C. C., and A. Tarski, "The algebra of topology," Annals of Mathematics, vol. 45, ser. 2 (1944), pp. 141-151.

[6] Rasiowa, H., and R. Sikorski, The Mathematics of Metamathematics, Monografie Matematyczne, tom 41, Państwowe Wydawnictwo Naukowe, Warszawa (1963).

[7] Sobociński, B., "Solution to the problem concerning the Boolean bases for cylindric algebras," Notre Dame Journal of Formal Logic, vol. XIII (1972), pp. 529545 .

University of Notre Dame

Notre Dame, Indiana 